\section{Evaluation of C-C Chemokine Ligand 5 (CCL5) Chemokine, Interleukin 5 (IL-5) Cytokine, and Eosinophil Counts as Potential Biomarkers in Saudi Patients with Chronic Asthma During Sandstorms}

\author{
Wael H. Alturaiki ${ }^{1}$ \\ SAU

\section{Abstract \\ Background and objectives}

1. Department of Medical Laboratory Sciences, College of Applied Medical Sciences, Majmaah University, Majmaah,

Corresponding author: Wael H. Alturaiki, w.alturaiki@mu.edu.sa

Asthma is a common chronic inflammatory disorder of the lung that can be exacerbated by environmental triggers during sandstorms. This study aimed to evaluate the usefulness of C-C chemokine ligand 5 (CCL5) chemokine and interleukin 5 (IL-5) cytokine and determine the total eosinophil count in blood and sputum for use as biomarkers in Saudi patients with chronic asthma who visited emergency departments during sandstorms.

\section{Methods}

The study included 42 Saudi patients with chronic asthma and 20 healthy controls. Plasma levels of CCL5, IL-5, and total immunoglobulin E (IgE) were measured using a specific enzyme-linked immunosorbent assay (ELISA). Total eosinophils in peripheral blood were counted using a hematology analyzer (CELL-DYN Ruby System; Abbott Diagnostics, Chicago, Illinois); in sputum, eosinophils stained with Giemsa were examined under a microscope, counted, and expressed as a percentage of the total cells.

\section{Results}

Total IgE levels were significantly higher in patients with asthma (mean $433 \mathrm{IU} / \mathrm{ml}, \mathrm{P}=0.0001$ ) as compared to normal controls $(139 \mathrm{IU} / \mathrm{ml})$. There was no significant difference in the levels of CCL5 in patients with asthma $(625 \mathrm{pg} / \mathrm{ml})$ as compared to normal controls $(663 \mathrm{pg} / \mathrm{ml}, \mathrm{P}=0.57)$. No correlation was found between total IgE and CCL5 levels. IL-5 was not detected in patients with asthma or in controls. Moreover, the total counts of eosinophils in the blood did not increase in patients with asthma as compared to controls while eosinophils in sputum samples were increased in the former (mean =3.128\%).

Received 03/18/2020

Review began 03/23/2020 Review ended 04/17/2020 Published 04/24/2020

(c) Copyright 2020 Alturaiki. This is an open access article distributed under the terms of the Creative Commons Attribution License CC-BY 4.0., which permits unrestricted use, distribution, and reproduction in any medium, provided the original author and source are credited.

\section{Conclusion}

Plasma levels of CCL5 and IL-5 or eosinophil counts in the peripheral blood may not be useful diagnostic biomarkers to evaluate airway inflammation and monitor asthma severity. Conversely, the sputum eosinophil count may represent a useful diagnostic marker for assessing the magnitude of asthma exacerbation during sandstorms.

Categories: Allergy/Immunology, Environmental Health

Keywords: asthma, sandstorms, total ige, ccl5, il-5, eosinophil

\section{Introduction}

Asthma is a common chronic inflammatory disorder of the lung characterized by bronchial hyperresponsiveness with clinical symptoms, including wheezing, coughing, mucus production, migration of inflammatory immune cells into airways, and shortness of breath [1]. According to the World Health Organization (WHO), approximately 235 million individuals worldwide have asthma [2]. Asthma is considered one of the most common chronic diseases in Saudi Arabia, affecting more than 2 million people over the past three decades, possibly as a result of changes in lifestyle, dietary habits, and exposure to environmental factors such as dust, indoor allergens, tobacco, and sandstorms [3].

Major sandstorms are common in the Middle East [4]. During these sandstorms, patients with asthma are at a very high risk of disease exacerbation. Acute asthma exacerbations are considered one of the most common reasons for emergency room visits, and such exacerbation can be initiated by exposure to dust or during sandstorms [5-6]. These sandstorms carry some of the most important allergenic fungal spores that may 
Atopic asthma is classified as a type-1 hypersensitivity, where immunoglobulin E (IgE) has a crucial function in the allergic reaction [8]. Eosinophils play a key role in asthma, and these cells have been shown to contribute to disease exacerbation [9]. Accordingly, there is a need for better markers of disease exacerbation in patients exposed to sandstorms.

Regulated upon activation, normal T-cell expressed and secreted (RANTES), a chemokine also known as C-C chemokine ligand 5 (CCL5), is a member of the CC chemokine family with a molecular weight of $7.5 \mathrm{kDa}$ that plays an important role in the inflammatory process [10]. CCL5 is a potent leucocyte chemoattractant that upon interaction with its receptors, CCR1, CCR3, and CCR5, induces the activation and trafficking of a wide range of immune cells, including T-cells, monocytes, basophils, eosinophils, natural killer (NK) cells, and dendritic cells (DCs), to the site of inflammation [10]. Increased levels of CCL5 have been reported in patients with asthma [10-11]. Furthermore, targeting CCL5 with antibodies in a murine model of allergic airway disease was shown to inhibit airway inflammation [12].

Interleukin 5 (IL-5) is a Th2 cytokine with a molecular weight of approximately $52 \mathrm{kDa}$ that has also been shown to play an important role in the pathogenicity of asthma. IL-5 interacts with its receptor (IL-5Ra) expressed on eosinophils, maintaining airway inflammation and resulting in worsened asthma symptoms. Targeting IL-5 or its receptor has been shown to be a promising therapeutic approach for severe asthma [13]. Based on the above findings, CCL5, IL-5, and eosinophils clearly play a key role in the development of asthma.

In this study, the plasma levels of the chemokine CCL5 and cytokine IL-5 and total IgE and eosinophil counts in the peripheral blood and sputum were investigated in patients with asthma and normal controls during sandstorms to assess their possible use as biomarkers to evaluate disease exacerbation in patients exposed to sandstorms.

\section{Materials And Methods \\ Subjects}

Forty-two people with allergic asthma (mean age 37.06 years) and 20 normal controls (mean age 31.08 years) were enrolled in this study. The characteristics of the subjects with asthma are shown in Table 1. Asthma was diagnosed according to the Saudi Initiative for Asthma guidelines [14]. The patients were undergoing treatment, including inhaled corticosteroids (ICS) with or without a long-acting beta-agonist (LABA). Samples were collected from patients with chronic symptoms of asthma who visited hospital emergency departments during sandstorms between April 2016 and May 2017. All patients with asthma were examined, and their clinical status was determined by specialty physicians at Al Zulfi General Hospital, Kingdom of Saudi Arabia. All patients with asthma included in this study were non-smokers or had no respiratory tract infection prior to and during the study. The normal controls were recruited and selected from healthy blood donors from the blood bank at Al Zulfi General Hospital and were defined as those who had no history of any allergic diseases and no signs of wheezing, did not smoke or have any other chronic diseases, respiratory infection, or recent vaccinations, and were not receiving treatment. The study was approved by the Majmaah University Ethical Committee (approval no. MUREC-April.01/COM-206), and written informed consent was obtained from all subjects. 


\section{Cureus}

\begin{tabular}{|c|c|c|}
\hline Categories & Control subjects & Subjects with asthma \\
\hline Number of subjects & 20 & 42 \\
\hline Sex (male/female) & $11 / 9$ & $23 / 19$ \\
\hline Mean age & 31.08 & 37.06 \\
\hline Duration of asthma (years) & - & 1 to $>30$ \\
\hline ICS usage LABA usage & & 366 \\
\hline \multicolumn{3}{|l|}{ Cinical presentation at the time of samping } \\
\hline Shortness of breath & No & Increased \\
\hline Cough & A symptomatic coughing & Continuing \\
\hline Wheezing & Normal & Frequently loud \\
\hline Chest tightness & No & Present \\
\hline Mucus production & Not present & Increased \\
\hline Respiratory tract infection (viral, bacterial, and fungal) & Not present & Not present \\
\hline Speaking & Normal & Difficult \\
\hline Mean of heart rate per minute & 96.06 & 109.07 \\
\hline Mean of $\mathrm{O}_{2}$ saturation $\%$ & 95.08 & 90.5 \\
\hline
\end{tabular}

\section{TABLE 1: Characteristics of subjects}

ICS: Inhaled corticosteroid; LABA: Long-acting beta-agonist

\section{Methods}

Blood Collection and Isolation

Peripheral blood was collected from healthy donors and patients. Following collection, the blood was transferred immediately to tubes containing the anticoagulant ethylenediaminetetraacetic acid (EDTA). The blood was centrifuged at $1800 \mathrm{rpm}$ for $5 \mathrm{~min}$ at room temperature. After centrifugation, the plasma aspirated and divided into aliquots in small plastic tubes.

Eosinophil Counts in Peripheral Blood and Sputum

After collection in EDTA tubes, a hematology analyzer (CELL-DYN Ruby System; Abbott Diagnostics, Chicago, Illinois) was used to determine the complete blood counts (CBCs) and differential counts of white blood cells (WBCs), including eosinophils. Blood eosinophilia was identified at a cutoff of $5 \%$ and/or $>300$ cells $/ \mathrm{mm}^{3}$. The sputum samples were processed as follows. Briefly, all study subjects were requested to cough sputum into plastic containers. The sputum samples were examined macroscopically to ensure that they were free of salivary contaminants. Satisfactory sputum samples were treated with dithiothreitol (1\% Dithiothreitol (DTT)) in phosphate-buffered saline for 20 min to homogenize and disrupt the disulfide bonds in the mucous and disperse the cells. The suspension was centrifuged, and the cell pellet was resuspended, transferred, and distributed thinly and evenly over a microscope slide. Giemsa staining was performed, and the number of eosinophils was assessed under a microscope. The eosinophil count is expressed as a percentage (\%). A sputum eosinophil count $\geqslant 2 \%$ of total leukocytes in a sputum sample was considered abnormal based on the Saudi Initiative for Asthma guidelines (2019) [14].

\section{Cytokine Measurement by ELISA}

The plasma obtained from the healthy controls and patients was used for measuring protein concentrations using specific ELISAs. Total IgE levels were measured using Quantikine ELISAs (Abcam, Cambridge, UK, cat\# ab108650). Human RANTES or CCL5 (R\&D, UK, cat\# DRN00B) and IL-5 (R\&D, UK, cat\# D5000B) levels were also measured using Quantikine ELISAs. The assays were conducted as described in the manufacturer's instructions for each kit. The optical density (OD) of each well at $450 \mathrm{~nm}$ was determined using a microplate reader (BioTek ELx800 Absorbance Microplate Reader; BioTek Company, Winooski, Vermont). KC Junior 


\section{Cureus}

software (BioTek) was used to determine the final protein concentrations.

\section{Statistics}

Data are expressed as mean \pm standard deviation (SD), and an independent nonparametric sample test (Mann-Whitney U test) was employed to evaluate statistical significance using GraphPad Prism 6 software (GraphPad Software, San Diego, California). Pearson's test was applied to evaluate correlations. P-values $<0.05$ indicate significance.

\section{Results}

Total IgE was significantly increased in patients with asthma as compared to normal controls

Atopic asthma is an allergic disease that is characterized by increased levels of IgE. To ensure that the samples received from the hospital were from patients with allergic asthma, plasma IgE levels were measured using an ELISA. Total IgE levels were significantly elevated in all patients with asthma (mean $433 \mathrm{IU} / \mathrm{ml}$ ) $(\mathrm{P}=0.0001)$ as compared to normal controls $(139 \mathrm{IU} / \mathrm{ml})$ (Figure 1).

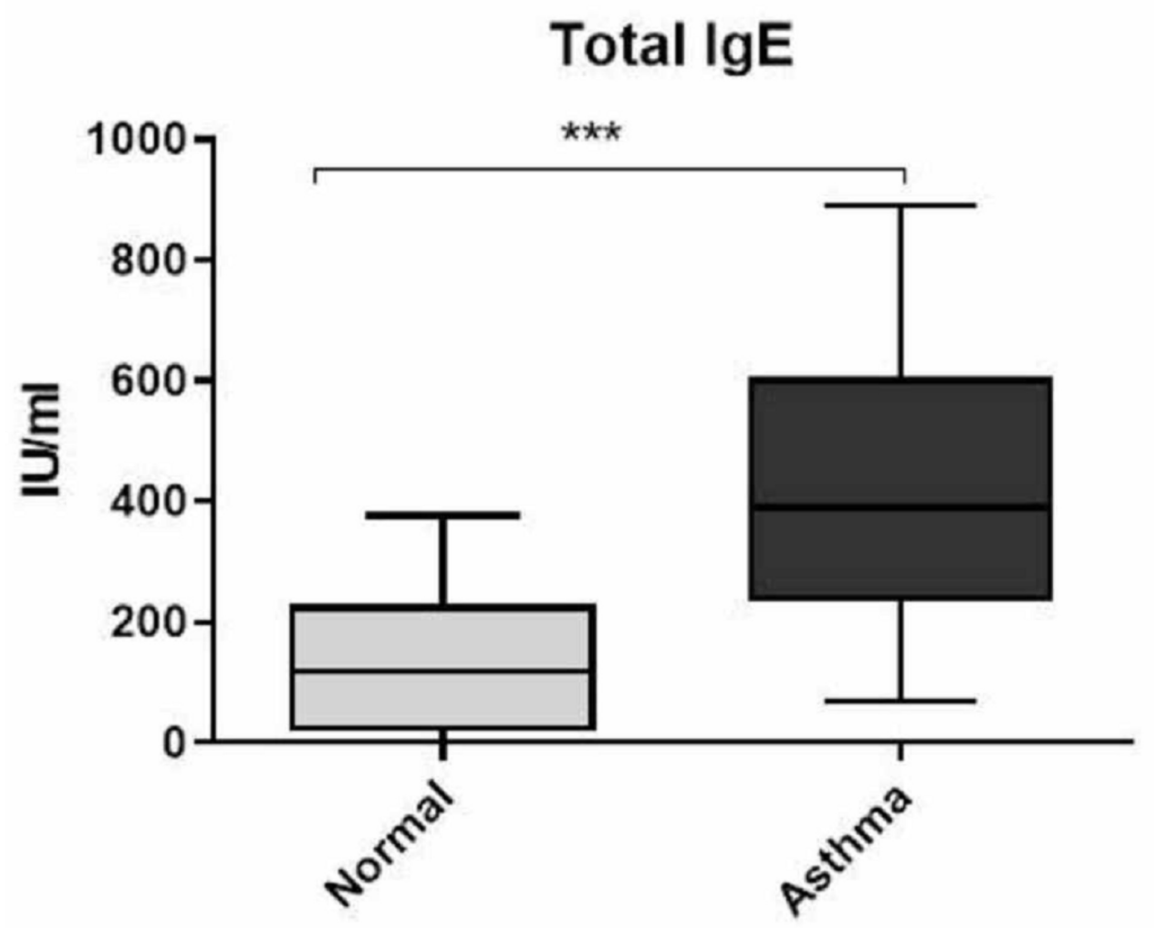

\section{FIGURE 1: Total IgE levels in the plasma}

Total IgE levels were measured using a specific ELISA. Total IgE levels were significantly elevated in patients with asthma compared to normal controls. Data are expressed as the mean \pm standard deviation (SD). ***P<0.0001.

IgE: immunoglobulin E; ELISA: enzyme-linked immunosorbent assay

\section{CCL5 chemokine protein levels were not significantly increased in patients with asthma as compared to normal controls}

No significant difference was observed in the levels of CCL5 chemokine between the patients ( $625 \mathrm{pg} / \mathrm{ml})$ and normal controls $(663 \mathrm{pg} / \mathrm{ml})(\mathrm{P}=0.57)$ (Figure 2A). Furthermore, no correlation was found between CCL5 and total IgE in the plasma of patients with asthma (Figure $2 B$ ). 


\section{Cureus}

A

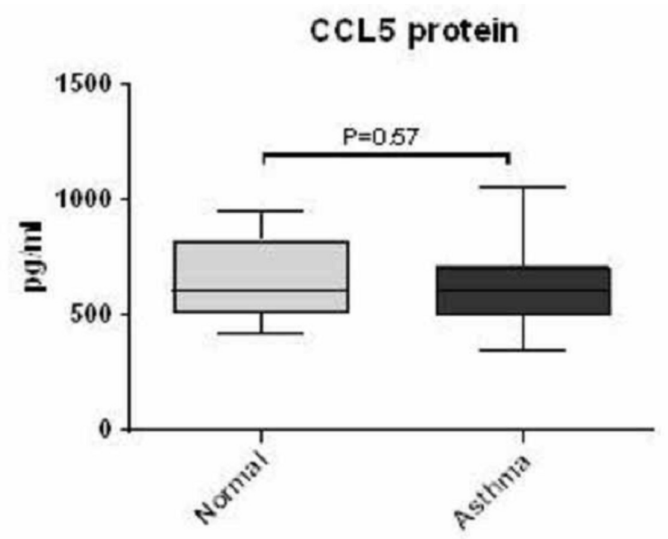

B

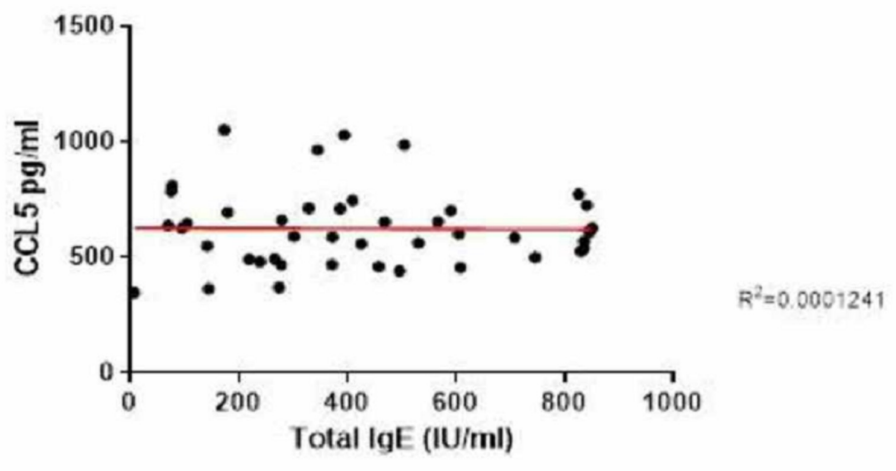

FIGURE 2: Plasma levels of CCL5 chemokine and correlation with total $\lg \mathrm{E}$

CCL5 chemokine levels were measured using a specific ELISA. No significant increase in CCL5 protein levels was observed in patients with asthma as compared to normal controls (A). Data are expressed as mean \pm standard deviation (SD). ${ }^{*} \mathrm{P}<0.05$. No correlation was found between CCL5 and total IgE in the plasma of patients with asthma based on Pearson's test (B).

ELISA: enzyme-linked immunosorbent assay; IgE: immunoglobulin E

\section{IL-5 cytokine was not detected, and total eosinophil counts were not increased in blood but in the sputum}

IL-5 was not detected in the plasma of normal controls or patients with asthma. The eosinophil count in the peripheral blood of the patients was within the normal range (mean 2.55\%) and was not significantly different when compared to that of the normal controls (mean 2.81\%) (Figure 3). However, the eosinophil count in the sputum of the patients with asthma was higher (mean $=3.128 \%$ ) than $2 \%$ because this percentage was considered abnormal based on the Saudi Initiative for Asthma guidelines [14]. 


\section{Cureus}

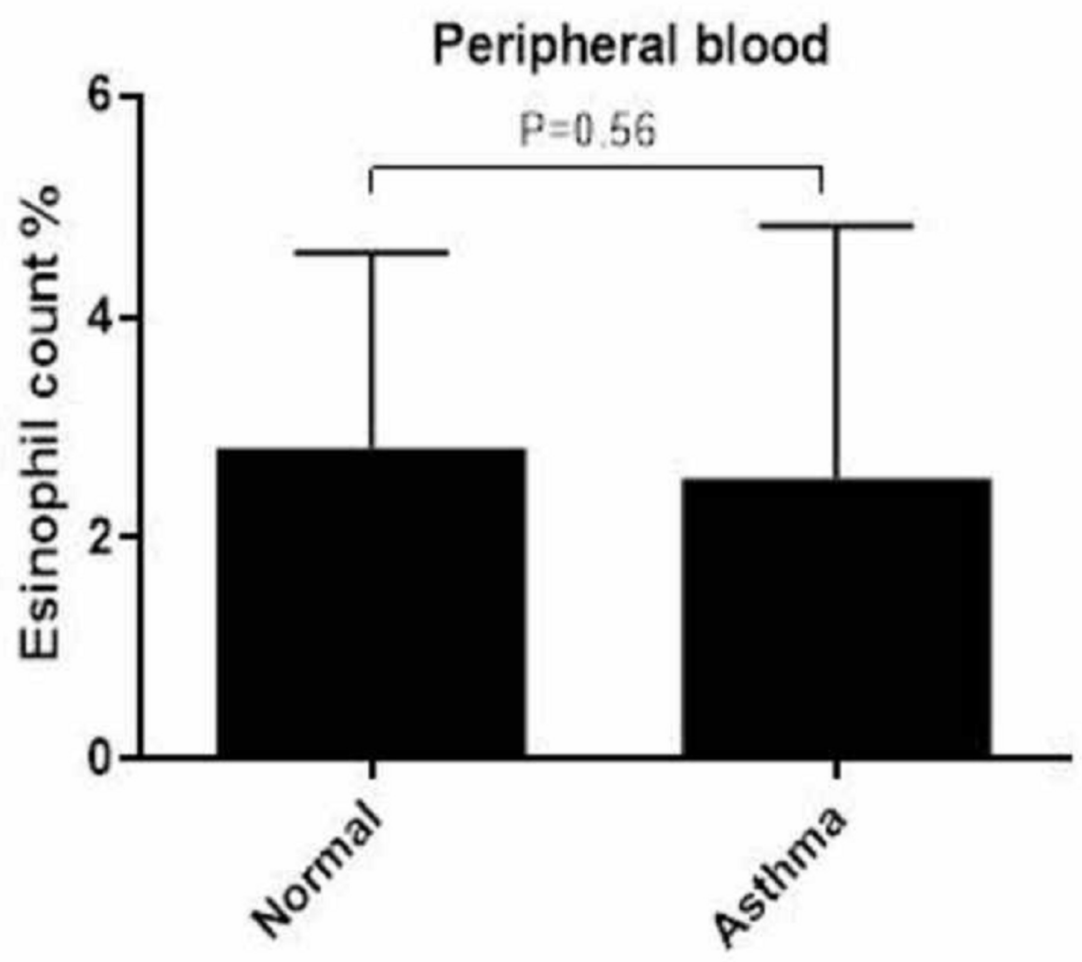

FIGURE 3: Eosinophil counts in the peripheral blood

No significant increase in eosinophils in the peripheral blood was observed in patients with asthma or normal controls. Data are expressed as mean \pm standard deviation (SD).

\section{Discussion}

This study aimed to evaluate plasma CCL5 (chemokine) and IL-5 (cytokine) as well as eosinophils in peripheral blood and sputum and their possible role in patients with chronic asthma who visited emergency rooms during sandstorms.

Unsurprisingly, a significant increase in total IgE was observed in patients with asthma (mean $433 \mathrm{IU} / \mathrm{ml}$ ) as compared to normal controls $(139 \mathrm{IU} / \mathrm{ml})$. The normal levels of total IgE values show a wide range of distribution. Total IgE levels ( $\geqslant 100 \mathrm{IU} / \mathrm{mL}$ ) have been used to determine atopic status [10]. However, in the Saudi population, it has been reported that $10 \%$ of individuals have values ( $\geqslant 200 \mathrm{IU} / \mathrm{ml}$ ) [15]. This is also in agreement with the current finding of the total IgE levels in the normal controls. This variation between the Saudi and Western populations may be explained as differences related to genetic, racial, and environmental factors [15].

In addition, there was no significant increase in CCL5 levels in the plasma of patients with asthma (mean $641 \mathrm{pg} / \mathrm{ml}$ ) relative to that in the healthy controls (588 pg/ml). Furthermore, no correlation was found between CCL5 and total IgE.

This finding is similar to that of Erten et al., who found no difference in CCL5 levels in patients with asthma relative to normal controls [16]. This finding may result from the inhaled corticosteroids used by these patients, which have been reported to suppress CCL5 via the inhibition of NF-KB-dependent transcription [17]. Other studies have also reported that CCL5 levels are significantly increased in the serum or plasma of patients with asthma who did not receive treatment prior to the study as compared to normal controls, with positive correlations with the severity score, total eosinophil count, and total serum IgE $[10,18]$. However, targeting the chemokine CCL5 with antibodies in a murine model of allergic airway disease resulted in the inhibition of airway inflammation, suggesting the importance of CCL5 in determining the severity of asthma [12].

Taken together, these results suggest that CCL5 may not be a useful marker of asthma severity during sandstorms, especially if the patients used ICS treatments during the storms. Furthermore, anti-CCL5 treatment would not be a useful therapeutic approach for this patient group. 
IL-5 plays a key role in eosinophil growth, differentiation, activation and effector function, and survival [19]. It has been reported that IL-5 levels in serum can be used as a biomarker for the blood eosinophilia asthma phenotype and that targeting IL-5 or its receptor alpha subunit (IL-5Ra) reduces eosinophil numbers and disease severity [20-21].

In this study, IL-5 was not detected in the plasma of patients with asthma or normal controls. The lower detection limit for IL-5 in the ELISA is $25 \mathrm{pg} / \mathrm{ml}$, and most studies have shown a range between $5 \mathrm{pg} / \mathrm{ml}$ and $20 \mathrm{pg} / \mathrm{ml}$, which is below the detection limit in the current study; therefore, the cytokine would not be detected. Moreover, treating asthma patients with ICS has been shown to suppress the transcription of the gene encoding IL-5 [22]. This finding suggests that IL-5 may not be a useful biomarker to monitor asthma progression if patients use ICS during sandstorms.

It has been proposed that blood eosinophil counts reflect the severity of asthma [23]. In addition, Durham et al. demonstrated the relationship between the number and action of blood eosinophils with the asthmatic response after allergen challenge, suggesting that elevated blood eosinophil counts correlate with airway inflammation in asthma [24]. Thus, blood eosinophil counts have been proposed as an indirect marker of airway inflammation in asthma [25]. Conversely, it has been shown that the total count of eosinophils in the blood cannot be used as a biomarker due to the variability of these cells in patients with asthma, which could not be explained by seasonality or diurnal variation [26-27]. This latter study is consistent with the findings of the current study, whereby no increase in eosinophil counts in the blood in patients with asthma relative to normal controls was observed. Again, these results can be attributed to the use of corticosteroids, which have been shown to inhibit eosinophils [28].

Interestingly, the total number of eosinophils in the sputum of patients with asthma patients was increased as compared to the normal range. Despite the importance of ICS in reducing sputum eosinophils and asthma exacerbations, the variability in response among patients with asthma must still be considered, as not all of these patients respond equally to ICS [29-30].

Taken together, the observations suggest that the eosinophil count in sputum, but not in blood, maybe a marker for predicting the severity of disease during asthma exacerbations and whether patients require higher doses of ICS to manage their asthma severity.

The limitations of the current study are that asthmatic patients with high plasma levels of total IgE were not examined for further evidence of allergic bronchopulmonary aspergillosis or parasitic infection. In addition, the analysis of CCL5 and IL-5 in the sputum was outside the scope of this work, and thus, consequent studies should investigate the probability that sputum cytokine expression is a good indicator of asthma activity.

\section{Conclusions}

In conclusion, the plasma levels of CCL5 and IL-5, as well as the eosinophil count in peripheral blood, may not be useful diagnostic biomarkers to evaluate airway inflammation and monitor asthma severity during sandstorms, especially if the patients have used ICS. However, the eosinophil count in the sputum may represent a useful diagnostic marker for asthma exacerbation.

\section{Additional Information \\ Disclosures}

Human subjects: Consent was obtained by all participants in this study. Majmaah University Ethical Committee issued approval MUREC-April.01/COM-206. The study was approved by the Majmaah University Ethical Committee (approval no. MUREC-April.01/COM-206), and written informed consent was obtained from all subjects. Animal subjects: All authors have confirmed that this study did not involve animal subjects or tissue. Conflicts of interest: In compliance with the ICMJE uniform disclosure form, all authors declare the following: Payment/services info: All authors have declared that no financial support was received from any organization for the submitted work. Financial relationships: All authors have declared that they have no financial relationships at present or within the previous three years with any organizations that might have an interest in the submitted work. Other relationships: All authors have declared that there are no other relationships or activities that could appear to have influenced the submitted work.

\section{Acknowledgements}

I would like to thank the deanship of scientific research at Majmaah University for supporting this work under project number (38/87). I would also like to express my gratitude to patients and healthy blood donors. In addition, I am deeply grateful to medical consultant Dr. Sajad Ahamed Mir and Dr. Adnan Afridi, as well as nursing staff and the head of the central laboratory and blood bank. I would like to express my deepest appreciation to the head of AlZulfi General Hospital. Special thanks to Dr. Suresh Mickymaray for unlimited support during the experiments and to Dr. Brian Flanagan for revising the manuscript. 


\section{References}

1. Kudo M, Ishigatsubo Y, Aoki I: Pathology of asthma. Front Microbiol. 2013, 4:263-263. 10.3389/fmicb.2013.00263

2. WHO. Asthma. Global prevalence. (2019). Accessed: November 24, 2019: https://www.who.int/newsroom/q-a-detail/asthma.

3. Al Ghobain MO, Algazlan SS, Oreibi TM: Asthma prevalence among adults in Saudi Arabia. Saudi Med J. 2018, 39:179-184.

4. Draxler RR, Gillette DA, Kirkpatrick JS, Heller J: Estimating PM10 air concentrations from dust storms in Iraq, Kuwait and Saudi Arabia. Atmos Environ. 2001, 35:4315-4330.

5. Tsai CL, Lee WY, Hanania NA, Camargo CA Jr: Age-related differences in clinical outcomes for acute asthma in the United States, 2006-2008. J Allergy Clin Immunol. 2012, 129:1252-1258. 10.1016/j.jaci.2012.01.061

6. Subbarao P, Mandhane PJ, Sears MR: Asthma: epidemiology, etiology and risk factors. CMAJ. 2009, 181:181190. 10.1503/cmaj.080612

7. Vijayakumar R, Aboody MSA, Alturaiki W, Alsagaby SA, Sandle T: A study of airborne fungal allergens in sandstorm dust in Al-Zulfi, central region of Saudi Arabia. J Environ Occup Sci. 2017, 6:27-33. 10.5455/jeos.20170120094512

8. Platts-Mills TAE: The role of immunoglobulin E in allergy and asthma . Am J Respir Crit Care. 2001, 164:1-5. 10.1164/ajrccm.164.supplement 1.2103024

9. Nakagome K, Nagata M: Involvement and possible role of eosinophils in asthma exacerbation . Front Immunol. 2018, 9:2220-2220. 10.3389/fimmu.2018.02220

10. Saad-El-Din Bessa S, Abo El-Magd GH, Mabrouk MM: Serum chemokines RANTES and monocyte chemoattractant protein-1 in Egyptian patients with atopic asthma: relationship to disease severity. Arch Med Res. 2012, 43:36-41. 10.1016/j.arcmed.2012.01.009

11. Alam R, York J, Boyars M, et al.: Increased MCP-1, RANTES, and MIP-1alpha in bronchoalveolar lavage fluid of allergic asthmatic patients. Am J Respir Crit Care. 1996, 153:1398-1404. 10.1164/ajrccm.153.4.8616572

12. Lukacs NW, Strieter RM, Warmington K, Lincoln P, Chensue SW, Kunkel SL: Differential recruitment of leukocyte populations and alteration of airway hyperreactivity by C-C family chemokines in allergic airway inflammation. J Immunol. 1997, 158:4398-4404.

13. Bagnasco D, Ferrando M, Varricchi G, Puggioni F, Passalacqua G, Canonica GW: Anti-interleukin 5 (IL-5) and IL-5Ra biological drugs: efficacy, safety, and future perspectives in severe eosinophilic asthma. Front Med. 2017, 4:135. 10.3389/fmed.2017.00135

14. Al-Moamary M, Alhaider S, Alangari A, et al.: The Saudi Initiative for Asthma - 2019 update: guidelines for the diagnosis and management of asthma in adults and children. Ann Thorac Med. 2019, 14:3-48. 10.4103/atm.ATM_327_18

15. Harfi HA, Godwin JT: Normal serum levels of IgG, IgA, IgM, IgD, and IgE in Saudi Arabia . Ann Saudi Med. 1985, 5:99-104. 10.5144/0256-4947.1985.99

16. Erten G, Muesellim B, Tutluoglu B, Gemicloglu B, Deniz G: Peripheral blood lymphocyte activation and RANTES levels in asthma. Turk J Med Sci. 2008, 38:531-536.

17. John M, Oltmanns U, Binder C, Meiners S, Gellert K, Chung KF, Witt C: Inhibition of chemokine production from human airway smooth muscle cells by fluticasone, budesonide and beclomethasone. Pulm Pharmacol Ther. 2004, 17:41-47. 10.1016/j.pupt.2003.09.002

18. Lun SW, Wong CK, Ko FW, Ip WK, Hui DS, Lam CW: Aberrant expression of CC and CXC chemokines and their receptors in patients with asthma. J Clin Immunol. 2006, 26:145-152. 10.1007/s10875-006-9003-9

19. Greenfeder S, Umland SP, Cuss FM, Chapman RW, Egan RW: Th2 cytokines and asthma. The role of interleukin-5 in allergic eosinophilic disease. Respir Res. 2001, 2:71-79. 10.1186/rr41

20. Agache I, Strasser DS, Klenk A, et al.: Serum IL-5 and IL-13 consistently serve as the best predictors for the blood eosinophilia phenotype in adult asthmatics. Allergy. 2016, 71:1192-1202. 10.1111/all.12906

21. Laviolette M, Gossage DL, Gauvreau G, et al.: Effects of benralizumab on airway eosinophils in asthmatic patients with sputum eosinophilia. J Allergy Clin Immunol. 2013, 132:1086-1096. 10.1016/j.jaci.2013.05.020

22. Barnes PJ: Inhaled corticosteroids. Pharmaceuticals. 2010, 3:514-540. 10.3390/ph3030514

23. Kamfar HZ, Koshak EE, Milaat WA: Is there a role for automated eosinophil count in asthma severity assessment?. J Asthma. 1999, 36:153-158. 10.3109/02770909909056311

24. Durham SR, Loegering DA, Dunnette S, Gleich GJ, Kay AB: Blood eosinophils and eosinophil-derived proteins in allergic asthma. J Allergy Clin Immunol. 1989, 84:931-936.

25. Koh YI, Choi S: Blood eosinophil counts for the prediction of the severity of exercise-induced bronchospasm in asthma. Respir Med. 2002, 96:120-125. 10.1053/rmed.2001.1238

26. Mathur SK, Fichtinger PS, Evans MD, Schwantes EA, Jarjour NN: Variability of blood eosinophil count as an asthma biomarker. Ann Allergy Asthma Immunol. 2016, 117:551-553. 10.1016/j.anai.2016.08.010

27. Spector SL, Tan RA: Is a single blood eosinophil count a reliable marker for "eosinophilic asthma?" . J Asthma. 2012, 49:807-810. 10.3109/02770903.2012.713428

28. Landolina NAC, Levi-Schaffer F: Eosinophils as a pharmacological target for the treatment of allergic diseases. Curr Opin Pharmacol. 2014, 17:71-80. 10.1016/j.coph.2014.07.014

29. Green RH, Brightling CE, McKenna S, et al.: Asthma exacerbations and sputum eosinophil counts: a randomised controlled trial. Lancet. 2002, 360:1715-1721. 10.1016/s0140-6736(02)11679-5

30. Szefler SJ, Martin RJ, King TS, et al.: Significant variability in response to inhaled corticosteroids for persistent asthma. J Allergy Clin Immunol. 2002, 109:410-418. 10.1067/mai.2002.122635 